\title{
Knockdown of zinc transporter ZIP5 (SLC39A5) expression significantly inhibits human esophageal cancer progression
}

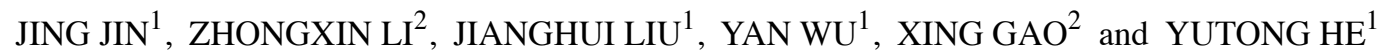 \\ ${ }^{1}$ Cancer Institute, ${ }^{2}$ General Surgery, Fourth Hospital of Hebei Medical University, Shijiazhuang, Hebei 050011, P.R. China
}

Received April 24,2015; Accepted May 26, 2015

DOI: 10.3892/or.2015.4097

\begin{abstract}
ZIP5 is a central player in mammalian zinc metabolism. Studies suggest thatZIP5 is differentially expressed during esophageal tumorigenesis, yet the role of ZIP5 in esophageal cancer cells has not yet been clarified. Immunohistochemistry, western blotting and qRT-PCR techniques were used to detect ZIP5 expression in esophageal squamous cell carcinoma (ESCC) tissues. We established a stable knockdown ZIP5 cell line (KYSE170K) derived from the ESCC cell line KYSE170. We conducted MTT and CCK-8 assays to determine the role of ZIP5 in cell proliferation, Transwell assays to detect migration and invasion, and flow cytometry (FCM) to detect apoptosis and cell cycle percentage using KYSE170K cells. We conducted a gene profiling study to detect the expression of genes related to tumor progression. The results demonstrated that ZIP5 protein and mRNA expression was highest in ESCC, intermediate in para-carcinoma and lowest in normal tissue. ZIP5 knockdown decreased proliferation by 28 and $38 \%$, respectively, according to the MTT and CCK- 8 assays. Migration and invasion decreased by 54 and $68 \%$, respectively, according to the Transwell assays. COX2 expression was decreased by 68 and $75 \%$ at the mRNA and protein level, respectively, and cyclin D1 mRNA and protein expression was decreased following 62 and $60 \%$, respectively, by knockdown of ZIP5, which upregulated the mRNA and protein expression of E-cadherin by 80 and 60\%, respectively. ZIP5 knockdown inhibited the proliferation, migration and invasion of ESCC and suppressed COX2, cyclin D1 and E-cadherin expression, which led to the inhibition of cell progression in ESCC.
\end{abstract}

\section{Introduction}

Esophageal cancer is one of the most common cancers in the world. With an estimated 456,000 new cases and 400,000 deaths, esophageal cancer was the eighth most common cancer

Correspondence to: Professor Yutong He, Cancer Institute, Fourth Hospital of Hebei Medical University, Shijiazhuang, Hebei 050011, P.R. China

E-mail: hytong69@yahoo.com

Key words: esophageal cancer, ZIP5, COX2, cyclin D1, E-cadherin and the sixth leading cause of cancer-related deaths worldwide in 2012. There are 223,000 new cases and 197,200 deaths in China, accounting for $52.8 \%$ of the incidence and $49.3 \%$ of the mortality from this disease worldwide (1). Although some progress has been made with multidisciplinary treatment in recent decades, the overall 5 -year survival rate is only $20 \%$ (2). There is a pressing need to understand more concerning esophageal cancer pathogenesis and to develop an effective treatment for esophageal cancer. It would be beneficial for therapeutics of esophageal cancer to target genes that are either more specifically expressed in esophageal cancer or involved in multiple pathways, such as cellular metabolism or nutrient uptake.

Zinc is an essential trace element and a catalytic/structural component that is used by many metalloenzymes and transcription factors. Zinc availability is also important for tumor growth and progression since zinc is a critical component for many enzymes that are involved in hypoxia, angiogenesis, cell proliferation and cancer metastasis. Most esophageal cancer cases arise in the rural countryside of China, which has limited health resources and an underdeveloped economy. Zou et al reported that a low nutrient intake was found for zinc, with only $72 \%$ of the recommended daily allowance (RDA) and 62\% of the RDA in spring and autumn, respectively, in high-risk areas (3), which may be one of the high-risk factors of esophageal cancer. Zinc and zinc transporters have been shown to have correlations with cancer risk (4-9), although the role of zinc and zinc transporters in esophageal cancer progression is largely unknown. Wu et al found that ZIP6 plays an important role in the prognosis of esophageal squamous cell carcinoma (ESCC), with a higher expression of ZIP6 protein being correlated with a shorter length of survival in individuals with advanced ESCC, and that knockdown of ZIP6 expression suppressed the proliferation and invasion of ESCC cells (7).

ZIP5 plays an important role in maintaining cellular zinc levels by facilitating the uptake of dietary zinc into intestinal epithelial cells and releasing zinc from vesicular compartments. It is primarily expressed in the liver, kidney, pancreas and small intestine but not in the esophagus $(10,11)$. Kumar et al found that ZIP5 was differentially expressed during esophageal tumorigenesis (12). ZIP5 and ZIP6 have very similar sequences, and they may have various similarities in terms of function. We hypothesized that ZIP5 plays a role in the development of esophageal cancer. 
In the present study, we detected the expression of ZIP5 in human esophageal tissues and esophageal cancer cell lines. A stable ZIP5-silenced cell line was established from the esophageal cancer cell line KYSE170 by short hairpin RNA using lentiviral vectors; we then compared the cell proliferation, migration and apoptosis of the esophageal cancer cell lines to discover the role of ZIP5 in esophageal cancer progression. We explored the mechanisms by detecting the expression of significant genes related to tumor progression to discover new targets for testing or treatment.

\section{Materials and methods}

Human esophageal cancer tissue specimens. The present study was approved by the Institutional Human Ethics Committee of Hebei Medical University Fourth Hospital, and prior informed consent was obtained from all patients. All specimens were obtained and pathologically confirmed to be ESCC at the Hebei Medical University Fourth Hospital, and Tumor Hospital of Hebei Province. Hebei Province is located in northern China on the North China Plain; it surrounds, but does not govern, Beijing the capital of China. The 58 patients from whom the samples were obtained underwent curative resection without previous radiotherapy and chemotherapy between 2012 and 2014. The patients, 37 males and 21 females, ranged in age from 44 to 77 years (mean age, 51 years). There were 39 patients at the early stage and 19 with advanced cancer. Tumor sizes were divided as $\leq 5 \mathrm{~cm}(\mathrm{n}=47)$ and $>5 \mathrm{~cm}(\mathrm{n}=11)$. Cancer, para-carcinoma and normal tissues were collected from each patient. The para-carcinoma tissue was obtained $2 \mathrm{~cm}$ distant from the tumor margin, and the normal tissue was located at the farthest edge from the tumor margin in the surgically resection tissue. All samples were anonymously coded in accordance with local ethical guidelines.

Immunohistochemical (IHC) assay. Paraffin sections (4-mm thick) were deparaffinized and rehydrated, followed by treatment with 0.02 M EDTA buffer (pH 9.0; Gene Tech, USA). Then, the sections were immersed in $3 \% \mathrm{H}_{2} \mathrm{O}_{2}$ to quench endogenous peroxidase activity and blocked with $5 \%$ normal goat serum, followed by incubation with the monoclonal anti-ZIP5 antibody (1:150; Sigma, USA) overnight at $4^{\circ} \mathrm{C}$. The antibody was diluted in phosphate-buffered saline (PBS) buffer containing 5\% normal goat serum. The negative control for each slide was incubated with $5 \%$ normal goat serum without the anti-ZIP5 antibody. The sections were then incubated with HRP-conjugated anti-rabbit IgG (ZSGB-BIO, China) for $45 \mathrm{~min}$ at $37^{\circ} \mathrm{C}$ and revealed with diaminobenzidine tetrahydrochloride. The stained slides were scored by three pathologists who were unaware of the clinical diagnosis. The indices of ZIP5 labeling were implemented so that samples were scored according to the percentage and intensity scores of the positively stained tumor cells.

Western blotting. Cells were lysed with ice-cold lysis buffer (Keygen Biotech, China) for $30 \mathrm{~min}$ on ice. Cell lysates were then collected after centrifugation at 12,000 rpm for $5 \mathrm{~min}$ at $4^{\circ} \mathrm{C}$. Sixty micrograms of lysate protein was loaded, and the total cellular protein was separated by $12 \%$ SDS-PAGE and then transblotted overnight at $4^{\circ} \mathrm{C}$ onto PVDF membranes.
The membranes were incubated with antibody (anti-ZIP5, 1:1,500; anti-GAPDH, 1:2,500; both from Sigma) at $4^{\circ} \mathrm{C}$ overnight, washed three times with TBST with $0.1 \%$ Tween, and incubated with a horseradish peroxidase-linked secondary antibody $(1: 2,000)$ for $1 \mathrm{~h}$ at room temperature. The membranes were washed three times with TBST with $0.1 \%$ Tween, and the immunoreactive bands were detected using an Enhanced Chemiluminescent Plus reagent kit.

RNA isolation and real-time qPCR. Total RNA was extracted using TRIzol reagent according to the manufacturer's protocol (Invitrogen, USA). The cDNA was synthesized by reverse transcription. The mRNA levels of ZIP5 were analyzed by real-time reverse transcriptase PCR. Briefly, real-time PCR was performed using the GoTaq qPCR Master Mix kit. PCR included $100 \mathrm{nmol} / \mathrm{l}$ of each primer, diluted cDNA templates and the mix, and was run for 35 cycles at $95^{\circ} \mathrm{C}$ for $30 \mathrm{sec}, 58^{\circ} \mathrm{C}$ for $45 \mathrm{sec}$ and $72^{\circ} \mathrm{C}$ for $30 \mathrm{sec}$. PCR efficiency was examined by serially diluting the template cDNA, and the melting curve data were collected to determine PCR specificity. Each cDNA sample was run in triplicate, and the corresponding no-reverse transcriptase mRNA sample was included as a negative control. The $\beta$-actin primer was included in every plate to avoid sample variations. The relative mRNA level was presented as unit values of $2^{[\mathrm{Ct}(\beta \text {-actin) - Ct (gene of interest)] }}$. The sequence of the forward primer for ZIP5 used in the present study was 5'-CTCATGCTTGCCATAACC-3' and the sequence of the reverse primer was 5'-AATCCTATTGCTCCTACTGG-3'.

Cell lines. Human esophageal cancer cell line KYSE170 was obtained from the MD Anderson Cancer Center, USA and was cultured in RPMI-1640 medium with $10 \%$ fetal bovine serum (FBS) (both from Gibco, USA). The cell line Eca109 was maintained in our laboratory. The cell lines KYSE70, KYSE150, KYSE180, KYSE510, EC9706 and CaES17 were purchased from Sangon Biotech (China). The cell line HEK 293T was obtained from the State Key Laboratory of Natural and Biomimetic Drugs of Peking University in China and was cultured in Dulbecco's modified Eagle's medium (DMEM; Gibco) with 10\% FBS.

Lentiviral vector packages and stable transduction. The potential target sequences were found using Ambion online tools and retrieval from the GenBank, and the siRNA was designed according to general design principles. The plasmid containing the specific shRNA was inserted into the PSD31 plasmid to obtain the lentiviral vector lenti-plasmid. The lentiviral vectors were jointly transfected into $293 \mathrm{~T}$ cells to obtain the viral solution. The $293 \mathrm{~T}$ cells were cultured in DMEM with sodium pyruvate and $10 \%$ FBS and were transfected with $2 \mu \mathrm{g}$ of the shRNA-lenti-plasmid, $2 \mu \mathrm{g}$ of pMDL, $1 \mu \mathrm{g}$ of $\mathrm{pRSV}$ and $1 \mu \mathrm{g}$ of VSVG using the transfection reagent Lipofectamine 2000 (Invitrogen) for $24 \mathrm{~h}$. After the A medium (DMEM without sodium pyruvate with $3 \%$ fetal calf serum) was changed at $6 \mathrm{~h}$, the lentiviruses were harvested at $48 \mathrm{~h}$ and filtered with a 0.45 -mm filter. Experiments for stable lentivector transduction were performed as follows: KYSE170 cells were seeded in a 6-well cell culture dish and transduced with the lentiviruses for $24 \mathrm{~h}$. Selection was performed starting at $48 \mathrm{~h}$ using $0.6 \mu \mathrm{g} / \mathrm{ml}$ puromycin until all parental cells from a 
parallel experiment died. The viable cells were the stable esophageal cancer cell lines with knockdown of ZIP5. The sequence of the ZIP5 shRNA used in the present study was 5'-CCUGCUGAGCAGGAGCAGAACCAUUACCU-3'. The stable cell line expressing ZIP5 shRNA was named KYSE170K, and the stable cell line expressing the empty vector was named KYSE170S.

MTT and CCK-8 assays. Esophageal cancer cells and the cells with knockdown of ZIP5 were seeded in 96-well plates $\left(5 \times 10^{3}\right.$ cells/well). Cell proliferation was assessed at 12, 24, 36 and $48 \mathrm{~h}$. Then, $20 \mu \mathrm{l}$ of MTT $(0.5 \%)$ reagent was added to each well, incubated at $37^{\circ} \mathrm{C}$ and $5 \% \mathrm{CO}_{2}$ for $4 \mathrm{~h}$, and then transduced with $150 \mu \mathrm{l}$ of dimethylsulfoxide (DMSO). Absorbance was recorded at $450 \mathrm{~nm}$ with a universal microplate reader. CCK-8 was analyzed with a simple test in which $10 \mu \mathrm{l}$ of CCK-8 reagent was added to each well and incubated at $37^{\circ} \mathrm{C}$ and $5 \% \mathrm{CO}_{2}$ for $2-4 \mathrm{~h}$, and the absorbance was recorded at $450 \mathrm{~nm}$.

Transwell assays. For the Transwell migration assay, $2 \times 10^{4}$ cells were plated in the top chamber with a non-coated membrane (24-well insert; pore size, $8-\mu \mathrm{m}$; BD Biosciences). For the invasion assay, $2.5 \times 10^{4}$ cells were plated in the top chamber with a Matrigel-coated membrane (24-well insert; pore size, $8-\mu \mathrm{m})$. In both assays, the cells were plated in medium without serum, and medium supplemented with serum was used as a chemoattractant in the lower chamber. The cells were incubated for $24 \mathrm{~h}$, and cells that did not migrate or invade through the pores were gently removed by a cotton swab. Cells on the lower surface of the membrane were fixed and stained with Giemsa crystal violet solution and counted under light microscopy magnification.

Flow cytometry (FCM) assays. Cells were harvested and washed twice with FBS and then fixed in a $70 \%$ ethanol solution for $24 \mathrm{~h}$ at $4^{\circ} \mathrm{C}$. Then, the fixed cells were washed once with PBS and resuspended as a single-cell suspension. The suspension was incubated with $500 \mu 1$ of propidium iodide (PI) $(50 \mu \mathrm{g} / \mathrm{ml})$ for $30 \mathrm{~min}$ and tested by FCM according to standard procedures.

Statistical analysis. All statistical analyses were performed using SPSS 13.0 software (SPSS, Inc., USA). Quantitative results are shown as mean \pm SD. The Student-Newman-Keuls test was used for statistical analyses between the groups. $\mathrm{P}<0.05$ was considered to indicate a statistically significant result.

\section{Results}

ZIP5 is overexpressed in human esophageal cancer tissue specimens and cell lines. According to the IHC and western blotting results, ZIP5 protein was overexpressed in the human esophageal cancer tissue samples compared with levels in the para-carcinoma and normal samples. The IHC of ZIP5 protein in the tissues of the esophageal cancer samples $(n=58$ normal-para-carcinoma-cancer groups) was examined. In normal esophageal epithelia, ZIP5 labeling was weak, as only $6(10.3 \%)$ normal samples were stained. In the para-carcinoma samples, $30(51.7 \%)$ were stained $(\mathrm{P}<0.01)$. ZIP5 was highly expressed in the cancer samples with $53(91.4 \%)$ of the cancer samples showing strong immunoreactivity to human ZIP5 $\mathrm{Ab}(\mathrm{P}<0.01)$. Based on the percentage and intensity of the positively stained cells, the immunoreactive score for normal samples was $0.19 \pm 0.66$. The score for the para-carcinoma samples was $1.14 \pm 1.29(\mathrm{P}<0.01)$, and the score for the cancer samples was 4.64 $\pm 3.67(\mathrm{P}<0.01)$ (Fig. 1A-C).

The protein expression of ZIP5 was measured in 18 groups of samples by western blotting. Using the expression of normal tissue as a standard, the relative quantity was $5.87 \pm 0.20$ in cancer tissues $(\mathrm{P}<0.01)$ and 3.42 \pm 0.92 in the para-carcinoma tissues $(\mathrm{P}<0.01)$. The results were consistent with the preceding results, demonstrating that the expression of ZIP5 in esophageal cancer tissues was higher (Fig. 1D and E).

The mRNA expression of ZIP5 was also measured in 58 groups of tissues by quantitative RT-PCR (qRT-PCR). With the expression of normal tissue as a standard, the relative quantity was $14.83 \pm 4.63$ in the cancer tissues $(\mathrm{P}<0.01)$ and $4.17 \pm 0.79$ in the para-carcinoma tissues $(\mathrm{P}<0.01)$ (Fig. $1 \mathrm{~F}$ and $\mathrm{G})$.

We examined the mRNA expression of ZIP5 in eight types of esophageal cancer cell lines, and the expression levels were high in all with the expression levels in KYSE170 and KYSE180 being the highest (Fig. 1H).

Knockdown of ZIP5 inhibits the proliferation, migration and invasion of esophageal cancer cells. To study the potential functions of ZIP5 in esophageal cancer, the stable ZIP5 knockdown cell line (KYSE170K) was established from the esophageal cancer cell line KYSE170 using a lentiviral vector. Stable cells containing empty vectors were also established from the KYSE170 cells to serve as controls (KYSE170S).

ZIP5 mRNA was measured by qRT-PCR. With the expression of this mRNA in the corresponding cell line (KYSE170S) as a standard, the relative quantity was 0.29 in the KYSE170K cells $(\mathrm{P}<0.01)$ and the efficiency of knockdown of ZIP5 mRNA was $71 \%$ (Fig. 2A and B).

We measured ZIP5 protein expression by western blotting. Using the ZIP5 expression in the KYSE170S cell line as a standard, the relative quantity was 0.10 in the KYSE170K cells $(\mathrm{P}<0.01)$, and the efficiency of knockdown of ZIP5 protein expression was $90 \%$ (Fig. 2C and D).

We compared the proliferation of KYSE170S and KYSE170K cells using MTT and CCK- 8 assays to investigate whether the knockdown of ZIP5 affects the proliferation of esophageal cancer cells, to study the functions of ZIP5 and to determine the effects of silencing of ZIP5 in vitro. The proliferation of the KYSE170K cell line was dramatically decreased by $28 \%$ when compared with that of the KYSE170S cell line, according to the MTT assay results, and by $38 \%$ according to the CCK-8 experimental results (Fig. 3A and B).

To investigate whether knockdown of ZIP5 affects the migration and invasion of esophageal cancer cells, we performed Transwell assays. Knockdown of ZIP5 significantly decreased the cell migratory (Fig. 3C and D) and invasive (Fig. 3E and F) abilities of the KYSE170K cell line by 54 and $68 \%$, respectively, compared with these abilities in the KYSE170S cell line.

The results of the FCM analyses showed that there was no difference in apoptosis in the two cell lines. However, the percentage of G1 phase cells $(50 \%)$ was significantly higher in 
A
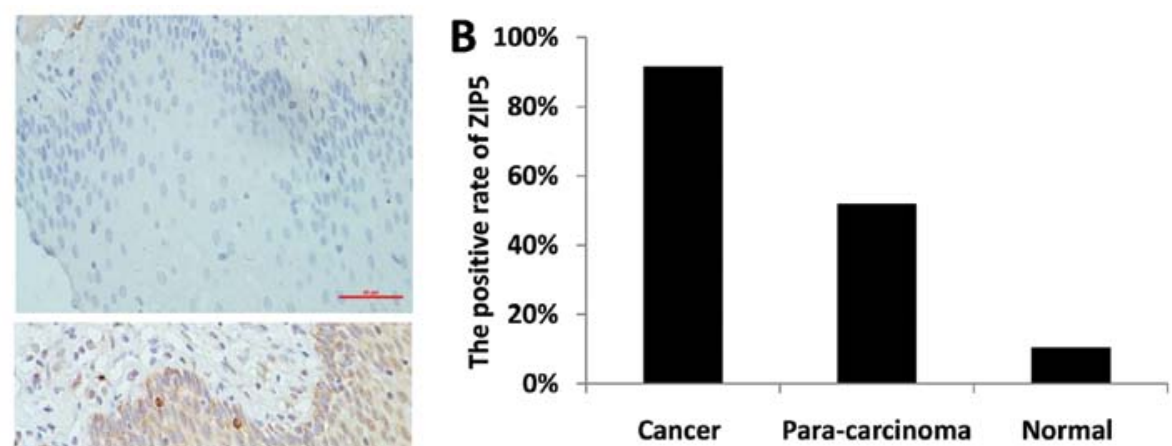

Para-carcinoma

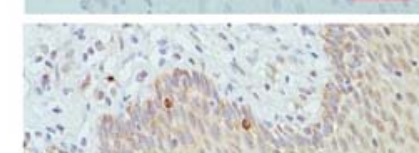

Cancer

Para-carcinoma Normal
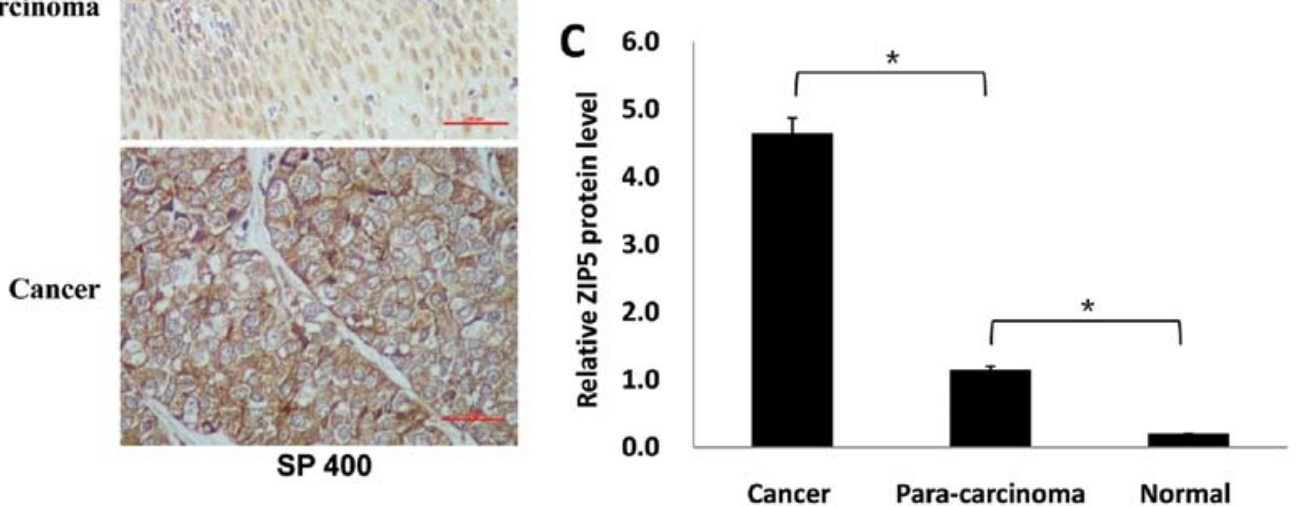

D
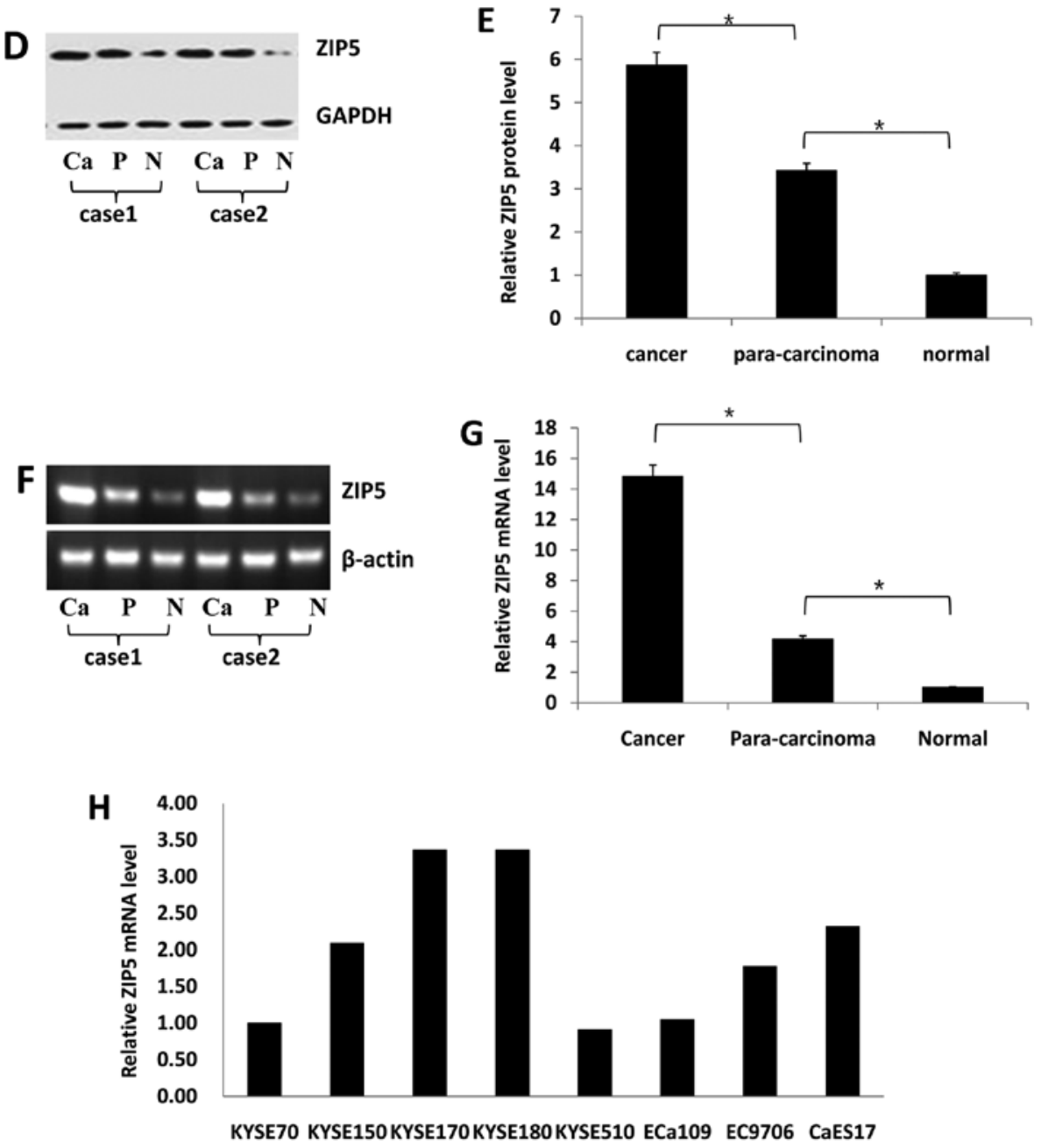

Figure 1. (A) Expression of ZIP5 in esophageal cancer tissues, para-carcinoma tissues and normal tissues was detected by IHC. (B) Positive rate of ZIP5 by IHC. (C) Protein expression levels of ZIP5 in esophageal cancer, para-carcinoma and normal tissues were detected by IHC. (D and E) Protein expression levels of ZIP5 in esophageal cancer, para-carcinoma and normal tissues were detected by western blotting ( $\left.{ }^{*} \mathrm{P}<0.01\right)$. The data are the means of the cases. (F and G) mRNA expression levels of ZIP5 in esophageal cancer, para-carcinoma and normal tissues were detected by qRT-PCR ("P<0.01). Data are the means of the cases. Ca, cancer; P, para-carcinoma; n, normal. (H) mRNA expression of ZIP5 in eight types of esophageal cancer cell lines. 
A

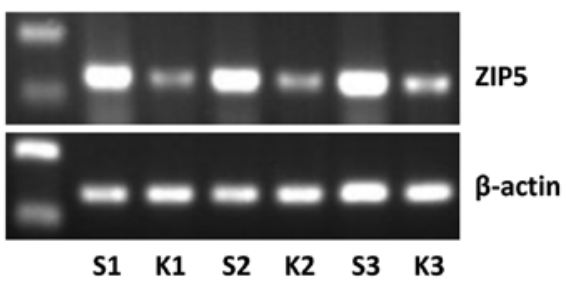

C

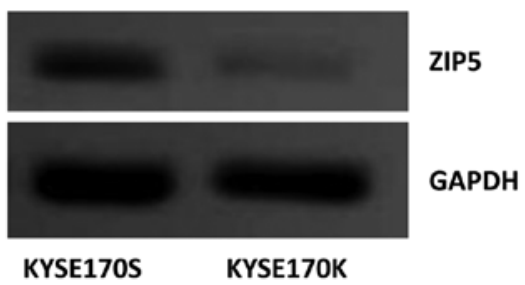

B
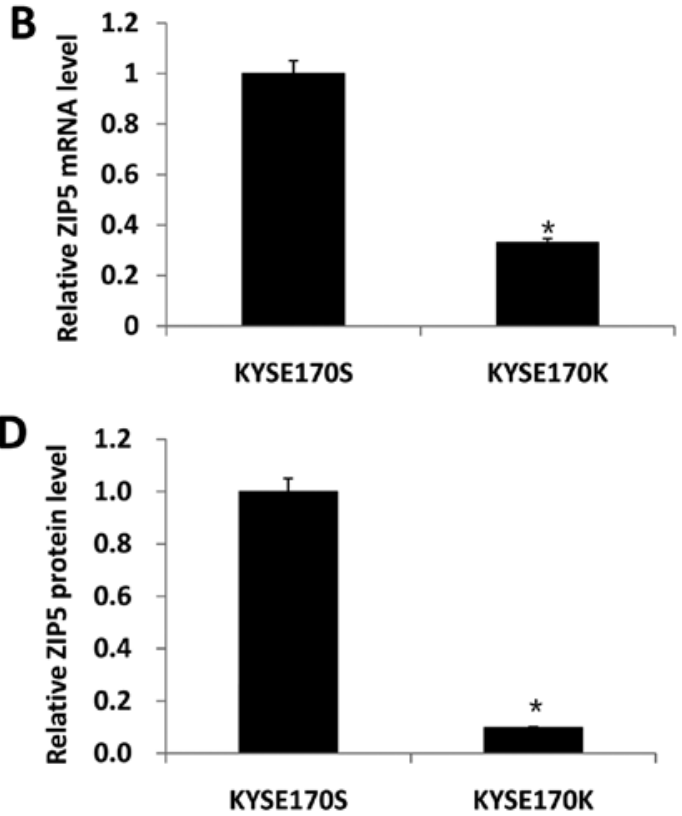

Figure 2. (A and B) Relative mRNA expression levels of KYSE170K cells were measured according to the standard mRNA expression value of KYSE170S cells, which was 1. (C and D) Relative protein expression levels of KYSE170K cells were measured according to the standard protein expression value of KYSE170S cells, which was 1 . $^{*} \mathrm{P}<0.01$.

the KYSE170K than that in the KYSE170S cells (25\%), and the difference was statistically significant (Fig. 3G).

Knockdown of ZIP5 expression inhibits the expression of COX2 and cyclin D1 and increases the expression of E-cadherin. We conducted a gene profiling study using the KYSE170S and KYSE170K cell lines to detect the expression of significant genes related to tumor progression, including COX2, cyclin D1, E-cadherin, bcl-2, bax, MMP-9, MMP-2, VEGF and p53, using qRT-PCR and western blotting. We found that the expression of COX2 and cyclin D1 was significantly downregulated in the KYSE170K cell line compared with the KYSE170S cell line, and the expression of E-cadherin was significantly upregulated in the KYSE170K cell line. We did not find differences in the expression levels of other genes (Fig. 4A).

Using the expression level in KYSE170S cells as a standard, the COX2 mRNA relative quantity was 0.32 in the KYSE170K cells $(\mathrm{P}<0.01)$. With the protein expression in KYSE170S cells as a standard, the relative quantity was 0.25 in the KYSE170K cells $(\mathrm{P}<0.01)$. Knockdown of ZIP5 suppressed $68 \%$ of COX2 at the mRNA level and $75 \%$ at the protein level (Fig. 4).

The mRNA expression level of cyclin D1 in the KYSE170K cells was 0.38 times the level in the KYSE170S cells according to $\mathrm{qRT}$-PCR $(\mathrm{P}<0.01)$. With the protein expression level in KYSE170S cells as a standard, the relative quantity was 0.40 in the KYSE170K cells $(\mathrm{P}<0.01)$. Knockdown of ZIP5 suppressed the expression level of cyclin D1 by $60 \%$ (Fig. 4).

The mRNA expression of E-cadherin in the KYSE170K cells was 1.8 times the expression in the KYSE170S cells according to $\mathrm{qRT}-\mathrm{PCR}(\mathrm{P}<0.01)$, and the protein expression level in KYSE170K cells was 1.6 times the expression in KYSE170S cells. Knockdown of ZIP5 expression upregulated the mRNA expression level of E-cadherin by $80 \%$ and the protein expression level by $60 \%$ (Fig. 4).

\section{Discussion}

Although the incidence of esophageal adenocarcinoma in Western countries has been rapidly increasing over the past few decades (13), the worldwide incidence of ESCC seems to be relatively stable or slightly decreased. Esophageal cancer is usually fatal, with an extremely poor 5-year survival rate of $20.9 \%$ in China (2). The main reason is that most cases are asymptomatic and go undetected until they are at an advanced stage and no longer amenable to surgical resection. It is important to determine the underlying mechanisms of esophageal cancer and search for new biomarkers for early detection and early treatment.

Zinc, an essential trace element, is necessary for the stabilization and function of numerous metalloenzymes involved in RNA and DNA and protein synthesis, and catabolism and energy metabolism (14). The intracellular zinc concentration is correlated to cell proliferation, differentiation and apoptosis $(15,16)$. As zinc treatments were first reported to inhibit tumor growth in animal models in 1969, there has been considerable interest in the role of zinc in cancer development and progression. In rodents, the association of zinc deficiency with the development of esophageal cancer has been unequivocally demonstrated, and differentially expressed zinc-regulated genes have been identified using microarrays (17-20). People who consume large quantities of whole grains and relatively little meat, which is a common dietary habit in high-risk areas are likely to be zinc deficient. Evidence from epidemiological studies suggests that zinc deficiency is associated with esophageal cancer (3). Abnet et al conducted a case-control study in a Chinese population that showed that a high tissue zinc concentration was strongly associated with a reduced risk of developing ESCC (21).

Zinc homeostasis is essential for maintaining normal physiological function. There are two important zinc transporter 

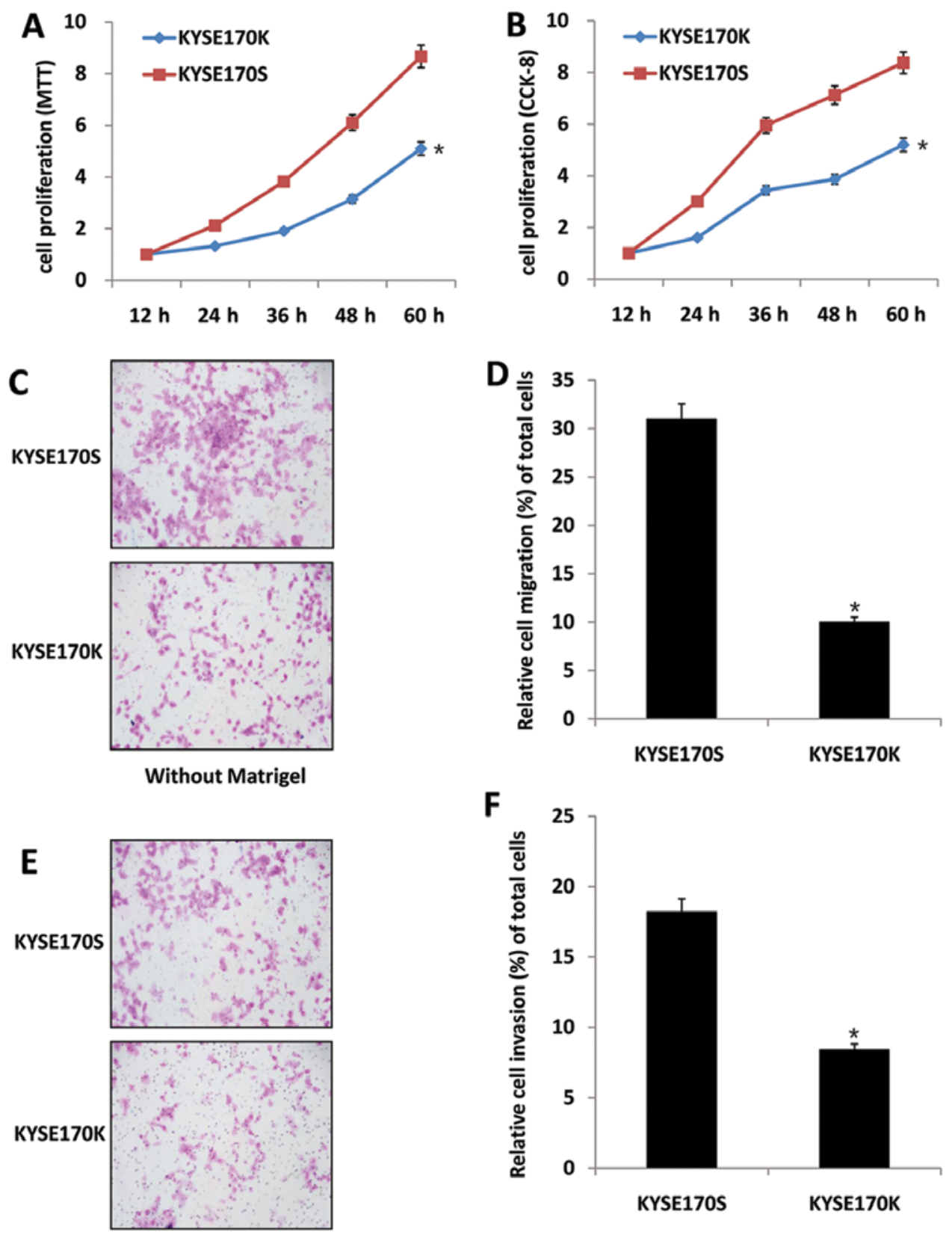

$\mathbf{F}$

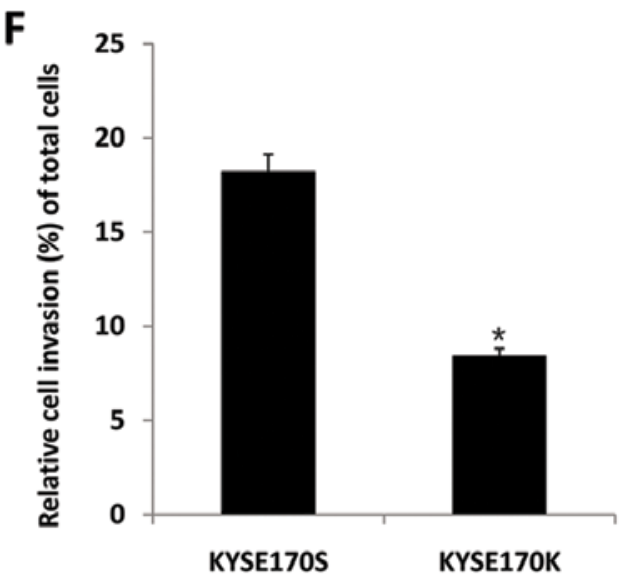

With Matrigel
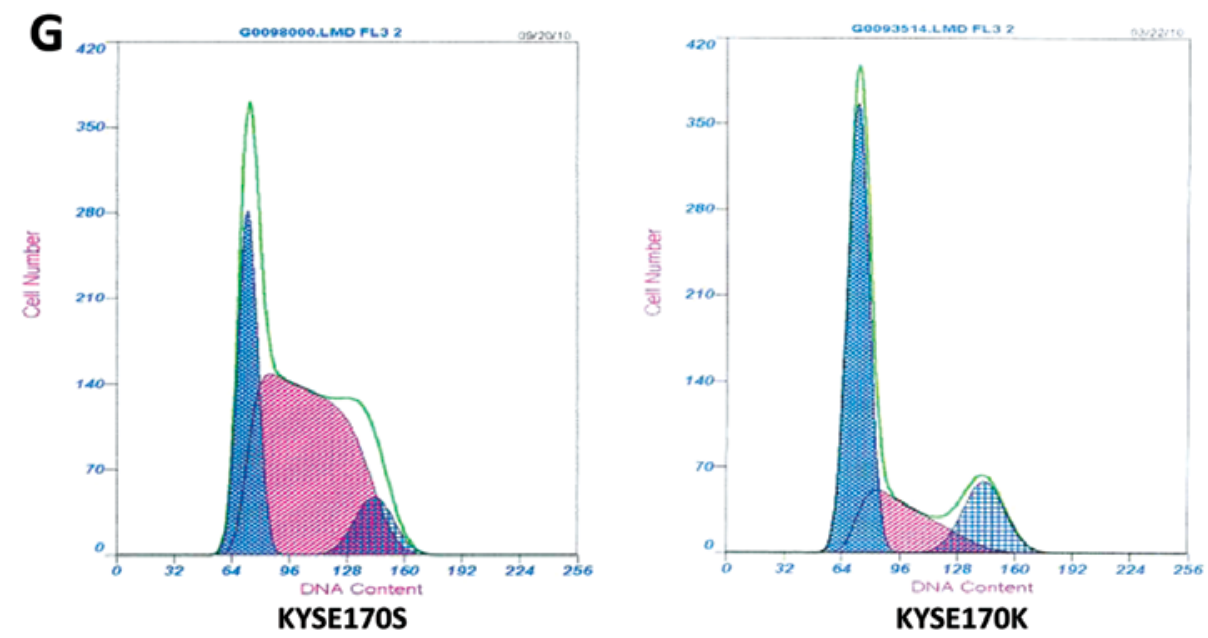

Figure 3. (A and B) Cell proliferation of KYSE170K cells was compared with that of KYSE170S cells using MTT and CCK-8 assays. (C and D) Cell migration of KYSE170K cells was compared with that of KYSE170S cells using a Transwell assay. (E and F) Cell invasion by KYSE170K cells was compared with that of KYSE170S cells using a Transwell assay. (G) Cell cycle percentage of KYSE170S and KYSE170K cells was detected by FCM analysis. FCM, flow cytometry. ${ }^{*} \mathrm{P}<0.01$. 

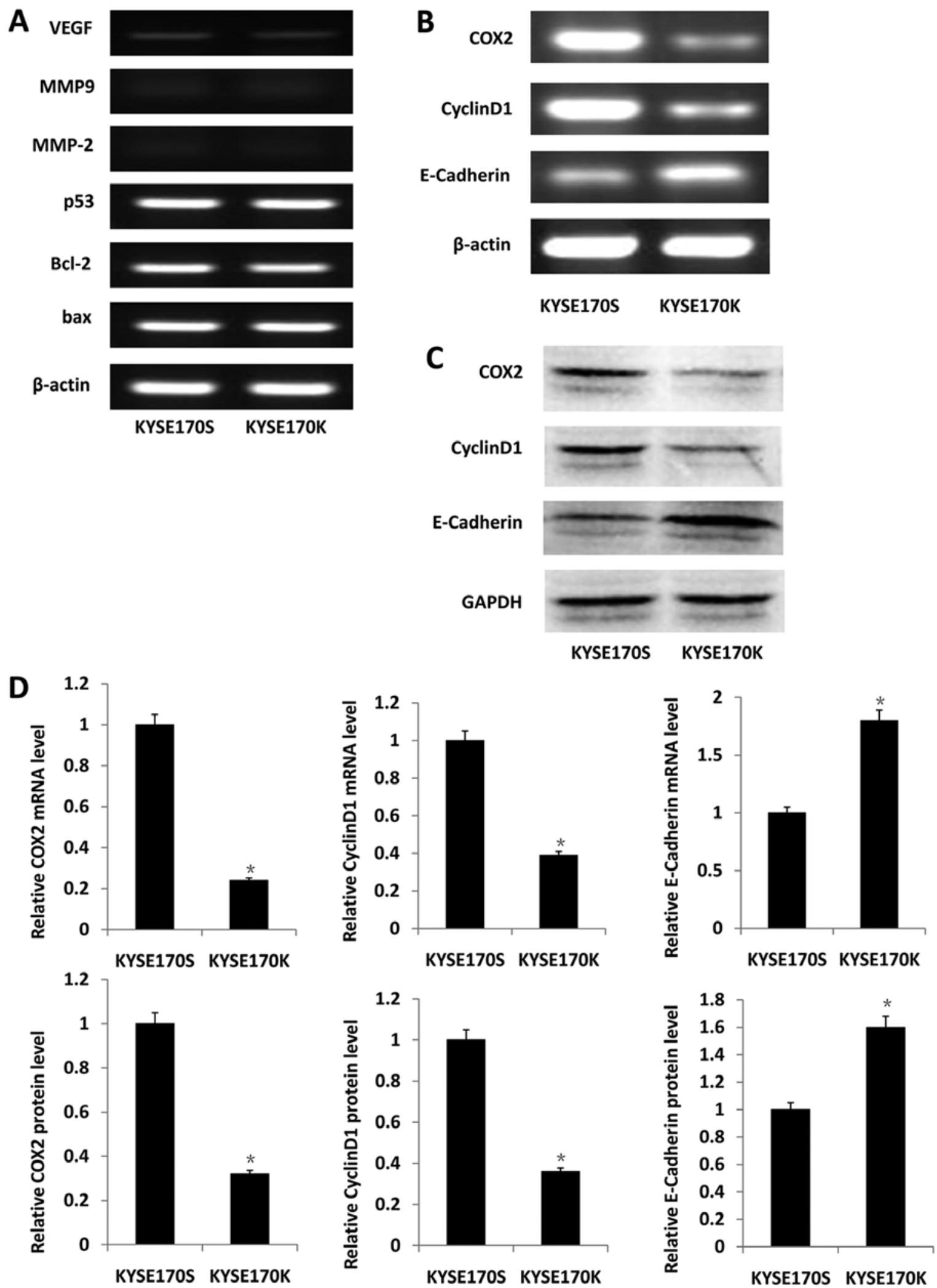

Figure 4. Detection of the expression of significant genes related to tumor progression in the stable cell lines using qRT-PCR and western blotting. (A) mRNA levels of VEGF, MMP-2, MMP-9, p53, bcl-2 and bax were analyzed by qRT-PCR and normalized to that of $\beta$-actin. (B) mRNA levels of COX2, cyclin D1 and E-cadherin were analyzed by qRT-PCR and normalized to that of $\beta$-actin. (C) Protein levels of COX2, cyclin D1 and E-cadherin were analyzed by western blotting and normalized to that of GAPDH. (D) Relative levels of COX2, cyclin D1 and E-cadherin were analyzed by qRT-PCR and western blotting. All data are the means of three separate experiments. ${ }^{*} \mathrm{P}<0.01$.

protein families that are directly involved in the metabolic homeostasis of intracellular zinc ions, ZIP and ZnT. The ZIP family contains ZIP1-ZIP14, and the ZnT family contains ZnT1-ZnT10; they are distributed in different tissues and 
organs $(9,14,22,23)$. The intracellular zinc level is determined by a variety of zinc transporter interactions, and the zinc transporter regulates intestinal zinc excretion to protect against zinc toxicity (7). ZIP5 is a central player in mammalian zinc metabolism and localizes to the basolateral surface of polarized cells. Some studies have shown that zinc transporters may play important roles in cancer progression. For example, the overexpression of ZIP1 promoted prostate cancer progression (4). Upregulation of ZIP4 was found to cause or accelerate pancreatic cancer (6). Increased expression of ZIP6 protein promoted proliferation and invasion in ESCC cells (7). The overexpression of ZIP7 and ZIP10 significantly increased the invasion of breast cancer cells $(8,9,24)$. Kumar et al presented a schematic illustration of different stages of esophageal tumorigenesis in India and identified 19 differentially expressed genes encoding zinc binding or modulating proteins, including zinc transporters ZnT7 and ZIP5 by microarray. They found that ZIP5 is affected by an upregulation of zinc metabolizing protein, suggesting the deregulation of zinc homeostasis during esophageal tumorigenesis (12), yet the relationship between ZIP5 and esophageal cancer has not yet been clarified.

The results of the present study illustrated that there was weak expression of ZIP5 in normal esophageal tissues, significantly increased expression in the para-cancerous tissues and significantly higher expression in cancer tissues. The high expression levels of ZIP5 corresponded to the degree of tumor malignancy; thus, ZIP5 plays an important role in the development of esophageal cancer. We found that the severity of hyperplasia and a high expression level of ZIP5 are consistent with other research studies on human esophageal cancer tissues, yet the causal relationship between them is not clear. Therefore, we established an esophageal cancer cell line with knockdown of ZIP5 expression to explore their relationship by researching the influence of the knockdown of ZIP5 on esophageal cancer cells. In this experiment, we used a lentiviral-mediated RNAi technology, and the silencing efficiency was $70 \%$ at the mRNA level and $90 \%$ at the protein level. We explored cell proliferation using MTT and CCK assays, and the results showed that the proliferation of the esophageal cancer cell lines was reduced significantly after ZIP5 knockdown. ZIP5 plays an important role in the proliferation of esophageal cancer cells. Silencing of ZIP5 was also associated with a decrease in cell migration and invasion. The results of the Transwell assays showed that the cell migratory and invasive abilities of the KYSE170K cells were decreased by 54 and $68 \%$, respectively, when compared with the KYSE170S cells which strongly suggests that ZIP5 not only regulates esophageal cancer cell proliferation but also has an impact on cancer metastasis.

We detected several target genes related to the proliferation, apoptosis, migration and invasion of cancer cells. COX2 is an important inducible enzyme during the development of the inflammatory reaction, and it is closely related to human tumors $(25,26)$. COX2 is overexpressed in a variety of tumors, particularly in digestive system cancer cells and cell lines (27-29). Upon examination of the COX2 expression of the stable cell lines, we found that the knockdown of ZIP5 suppressed the expression of COX2 in the esophageal cancer cells which may indicate that ZIP5 has an effect on the progression of esophageal cancer by influencing COX2. We also found that the cyclin D1 expression level in the KYSE170K cells was significantly lower than that in the KYSE170S cells. Cyclin D1 is a critical protein for the regulation of the G1 cell cycle phase, and its main function is to promote cell proliferation; hence, increased expression of cyclin D1 promotes cell proliferation. Therefore, we demonstrated that the knockdown of ZIP5 expression suppressed proliferation by reducing the expression levels of cyclin D1. The FCM results also supported the observation that increasing cells in the G1 phase following knockdown of ZIP5 weakened cell proliferation. Our result showed that the expression of E-cadherin was increased following knockdown of ZIP5. E-cadherin is a calcium-dependent cell adhesion molecule, and its main role is in regulating intercellular adhesion, and adhesion significantly inhibits the migration and invasion of tumors. Knockdown of ZIP5 upregulated the expression of E-cadherin, which explains the results in which the silencing of ZIP5 decreased cell migration and invasion.

In the present study, we explored the role of ZIP5 in esophageal cancer progression through cell experiments, and we will conduct more in vitro animal experiments to test and verify these results. We observed the phenomenon that ZIP5 was overexpressed in human esophageal cancer tissues but did not investigate whether zinc deficiency was the causen. These issues will be investigated in the future.

In summary, we discovered that the expression level of ZIP5 was highest in ESCC, intermediate in para-carcinoma and lowest in the normal tissue samples. We constructed a stable knockdown ZIP5 cell line and found that knockdown of ZIP5 expression decreased the proliferation, metastasis and invasion of esophageal cancer cells. These results establish an experimental foundation for further study and can help us to discover new therapies for esophageal cancer.

\section{Acknowledgements}

The present study was supported by grants from the National Natural Scientific Foundation of China (81272682), and the National Natural Scientific Foundation of Hebei Province (C2011206058).

\section{References}

1. GLOBOCAN 2012: Estimated Cancer Incidence, Mortality and Prevalence Worldwide in 2012. http://globocan.iarc.fr/Pages/ fact_sheets_cancer.aspx, 2012.

2. Zeng H, Zheng R, Guo Y, Zhang S, Zou X, Wang N, Zhang L, Tang J, Chen J, Wei K, et al: Cancer survival in China, 2003-2005: A population-based study. Int J Cancer 136: 1921-1930, 2015.

3. Zou XN, Taylor PR, Mark SD, Chao A, Wang W, Dawsey SM, Wu YP, Qiao YL and Zheng SF: Seasonal variation of food consumption and selected nutrient intake in Linxian, a high risk area for esophageal cancer in China. Int J Vitam Nutr Res 72: 375-382, 2002.

4. Makhov P, Golovine K, Uzzo RG, Wuestefeld T, Scoll BJ and Kolenko VM: Transcriptional regulation of the major zinc uptake protein hZip1 in prostate cancer cells. Gene 431: 39-46, 2009.

5. Iguchi K, Otsuka T, Usui S, Sugimura Y and Hirano K: Correlation between ZIP2 messenger RNA expression and zinc level in rat lateral prostate. Biol Trace Elem Res 112: 159-167, 2006.

6. Li M, Zhang Y, Liu Z, Bharadwaj U, Wang H, Wang X, Zhang S Liuzzi JP, Chang SM, Cousins RJ, et al: Aberrant expression of zinc transporter ZIP4 (SLC39A4) significantly contributes to human pancreatic cancer pathogenesis and progression. Proc Natl Acad Sci USA 104: 18636-18641, 2007.

7. Wu C, Li D, Jia W, Hu Z, Zhou Y, Yu D, Tong T, Wang M, Lin D, Qiao Y, et al: Genome-wide association study identifies common variants in SLC39A6 associated with length of survival in esophageal squamous-cell carcinoma. Nat Genet 45: 632-638, 2013. 
8. Taylor KM, Vichova P, Jordan N, Hiscox S, Hendley R and Nicholson RI: ZIP7-mediated intracellular zinc transport contributes to aberrant growth factor signaling in antihormoneresistant breast cancer Cells. Endocrinology 149: 4912-4920, 2008.

9. Hogstrand C, Kille P, Nicholson RI and Taylor KM: Zinc transporters and cancer: A potential role for ZIP7 as a hub for tyrosine kinase activation. Trends Mol Med 15: 101-111, 2009.

10. Wang F, Kim BE, Petris MJ and Eide DJ: The mammalian Zip5 protein is a zinc transporter that localizes to the basolateral surface of polarized cells. J Biol Chem 279: 51433-51441, 2004.

11. Dufner-Beattie J, Kuo YM, Gitschier J and Andrews GK: The adaptive response to dietary zinc in mice involves the differential cellular localization and zinc regulation of the zinc transporters ZIP4 and ZIP5. J Biol Chem 279: 49082-49090, 2004.

12. Kumar A, Chatopadhyay T, Raziuddin M and Ralhan R: Discovery of deregulation of zinc homeostasis and its associated genes in esophageal squamous cell carcinoma using cDNA microarray. Int J Cancer 120: 230-242, 2007.

13. Bosetti C, Levi F, Ferlay J, Garavello W, Lucchini F, Bertuccio P, Negri E and La Vecchia C: Trends in oesophageal cancer incidence and mortality in Europe. Int J Cancer 122: 1118-1129, 2008.

14. Gaither LA and Eide DJ: Eukaryotic zinc transporters and their regulation. Biometals 14: 251-270, 2001.

15. Franklin RB and Costello LC: Zinc as an anti-tumor agent in prostate cancer and in other cancers. Arch Biochem Biophys 463: 211-217, 2007.

16. Uzzo RG, Crispen PL, Golovine K, Makhov P, Horwitz EM and Kolenko VM: Diverse effects of zinc on NF-kappaB and AP-1 transcription factors: Implications for prostate cancer progression. Carcinogenesis 27: 1980-1990, 2006.

17. Fong LY, Sivak A and Newberne PM: Zinc deficiency and methylbenzylnitrosamine-induced esophageal cancer in rats. J Natl Cancer Inst 61: 145-150, 1978.

18. Fong LY, Lau KM, Huebner K and Magee PN: Induction of esophageal tumors in zinc-deficient rats by single low doses of $\mathrm{N}$-nitrosomethylbenzylamine (NMBA): Analysis of cell proliferation, and mutations in $\mathrm{H}-\mathrm{ras}$ and $p 53$ genes. Carcinogenesis 18 : $1477-1484,1997$.
19. Fong LY and Magee PN: Dietary zinc deficiency enhances esophageal cell proliferation and $N$-nitrosomethylbenzylamine (NMBA)-induced esophageal tumor incidence in C57BL/6 mouse. Cancer Lett 143: 63-69, 1999.

20. Fong LY, Nguyen VT and Farber JL: Esophageal cancer prevention in zinc-deficient rats: Rapid induction of apoptosis by replenishing zinc. J Natl Cancer Inst 93: 1525-1533, 2001.

21. Abnet CC, Lai B, Qiao YL, Vogt S, Luo XM, Taylor PR, Dong ZW, Mark SD and Dawsey SM: Zinc concentration in esophageal biopsy specimens measured by X-ray fluorescence and esophageal cancer risk. J Natl Cancer Inst 97: 301-306, 2005.

22. Palmiter RD and Findley SD: Cloning and functional characterization of a mammalian zinc transporter that confers resistance to zinc. EMBO J 14: 639-649, 1995.

23. Liuzzi JP and Cousins RJ: Mammalian zinc transporters. Annu Rev Nutr 24: 151-172, 2004

24. Kagara N, Tanaka N, Noguchi S and Hirano T: Zinc and its transporter ZIP10 are involved in invasive behavior of breast cancer cells. Cancer Sci 98: 692-697, 2007.

25. Song X, Lin HP, Johnson AJ, Tseng PH, Yang YT, Kulp SK and Chen CS: Cyclooxygenase-2, player or spectator in cyclooxygenase-2 inhibitor-induced apoptosis in prostate cancer cells. J Natl Cancer Inst 94: 585-591, 2002.

26. Fukazawa EM, Baiocchi G, Soares FA, Kumagai LY, Faloppa CC, Badiglian-Filho L, Coelho FR, Gonçalves WJ, Costa RL and Góes JC: Cox-2, EGFR, and ERBB-2 expression in cervical intraepithelial neoplasia and cervical cancer using an automated imaging system. Int J Gynecol Pathol 33: 225-234, 2014.

27. Dohadwala M, Luo J, Zhu L, Lin Y, Dougherty GJ, Sharma S Huang M, Pold M, Batra RK and Dubinett SM: Non-small cell lung cancer cyclooxygenase-2-dependent invasion is mediated by CD44. J Biol Chem 276: 20809-20812, 2001.

28. Costa C, Soares R, Reis-Filho JS, Leitão D, Amendoeira I and Schmitt FC: Cyclo-oxygenase 2 expression is associated with angiogenesis and lymph node metastasis in human breast cancer. J Clin Pathol 55: 429-434, 2002.

29. Smyth GP, Stapleton PP, Barden CB, Mestre JR, Freeman TA, Duff MD, Maddali S, Yan Z and Daly JM: Renal cell carcinoma induces prostaglandin E2 and T-helper type 2 cytokine production in peripheral blood mononuclear cells. Ann Surg Oncol 10: 455-462, 2003 